INTERAtional Electronic Journal of Algebra

VOLUME 29 (2021) 95-106

DOI: $10.24330 /$ ieja.852024

\title{
RM ALGEBRAS AND COMMUTATIVE MOONS
}

\author{
Andrzej Walendziak \\ Received: 24 January 2020; Revised: 27 April 2020; Accepted: 15 May 2020 \\ Communicated by Abdullah Harmancı

\begin{abstract}
Some generalizations of BCI algebras (the RM, BH, CI, BCH, $\mathrm{BH}^{* *}, \mathrm{BCH}^{* *}$, and ${ }^{*} \mathrm{RRM}^{* *}$ algebras $)$ satisfying the identity $(x \rightarrow 1) \rightarrow y=$ $(y \rightarrow 1) \rightarrow x$ are considered. The connections of these algebras and various generalizations of commutative groups (such as, for example, involutive commutative moons and commutative (weakly) goops) are described. In particular, it is proved that an RM algebra verifying this identity is equivalent to an involutive commutative moon.
\end{abstract}

Mathematics Subject Classification (2020): 03G25, 06A06, 06F35

Keywords: RM, BH, CI, BCH, BCI algebra, moon, p-semisimplicity

\section{Introduction}

In 1966, K. Iséki [6] introduced BCI algebras as algebraic models of BCI-logic. In 1983, Q. P. Hu and X. Li [2] defined BCH algebras, which are a generalization of BCI algebras. Later on, in 1998, Y. B. Jun et al. [7] introduced the notion of $\mathrm{BH}$ algebras. It is known that $\mathrm{BCI}$ and $\mathrm{BCH}$ algebras are contained in the class of BH algebras. Next, in 2009, the new class of algebras called CI algebras was introduced by B. L. Meng [11]. These algebras are a generalization of BCH algebras (hence also a generalization of BCI algebras). BH and CI algebras are also called aRM and RME algebras, respectively (see [3], [4], [14]). All of the algebras mentioned above are contained in the class of RM algebras (an RM algebra is an algebra $(A ; \rightarrow, 1)$ of type $(2,0)$ satisfying the identities: $x \rightarrow x=1$ and $1 \rightarrow x=x)$. Deductive systems and congruences in RM algebras were studied by A. Walendziak [12]. The implicative and commutative properties for some subclasses of the class of RM algebras were investigated in [13,14]. In 1985, T. Lei and C. Xi [9] defined psemisimple BCI algebras and proved that p-semisimple BCI algebras are equivalent with commutative groups. The p-semisimple BCI algebras have been extensively investigated in many papers (for example [1], [8], [10], [16]).

In this paper, we consider some generalizations of $\mathrm{BCI}$ algebras (the $\mathrm{RM}, \mathrm{BH}$, $\mathrm{CI}, \mathrm{BCH}, \mathrm{BH}^{* *}, \mathrm{BCH}^{* *}$, and ${ }^{*} \mathrm{aRM}^{* *}$ algebras) satisfying the identity $(x \rightarrow 1) \rightarrow$ $y=(y \rightarrow 1) \rightarrow x$, which plays a very important role in the theory of p-semisimple 
BCI algebras. We describe the connections of considered algebras and various generalizations of commutative groups (such as, for example, involutive commutative moons and commutative goops, which were introduced by A. Iorgulescu [5]). In particular, we prove that RM algebras verifying $(x \rightarrow 1) \rightarrow y=(y \rightarrow 1) \rightarrow x$ are equivalent with involutive commutative moons.

\section{Preliminaries}

\subsection{Generalizations of BCI algebras}

Let $\mathcal{A}=(A ; \rightarrow, 1)$ be an algebra of type $(2,0)$. We define the binary relation $\leqslant$ by: for all $x, y \in A$,

$$
x \leqslant y \Longleftrightarrow x \rightarrow y=1 .
$$

We consider the following list of properties (cf. [3]) that can be satisfied by $\mathcal{A}$ :

(An) (Antisymmetry) $x \rightarrow y=1=y \rightarrow x \Longrightarrow x=y$,

(An') (Antisymmetry) $x \leqslant y$ and $y \leqslant x \Longrightarrow x=y$,

(B) $(y \rightarrow z) \rightarrow[(x \rightarrow y) \rightarrow(x \rightarrow z)]=1$,

(B') $y \rightarrow z \leqslant(x \rightarrow y) \rightarrow(x \rightarrow z)$,

(BB) $(y \rightarrow z) \rightarrow[(z \rightarrow x) \rightarrow(y \rightarrow x)]=1$,

(BB') $y \rightarrow z \leqslant(z \rightarrow x) \rightarrow(y \rightarrow x)$,

(D1) $x \rightarrow((x \rightarrow 1) \rightarrow 1)=1$,

(D1') $x \leqslant(x \rightarrow 1) \rightarrow 1$,

(Ex) $x \rightarrow(y \rightarrow z)=y \rightarrow(x \rightarrow z)$,

(M) $1 \rightarrow x=x$,

(Re) (Reflexivity) $x \rightarrow x=1$,

(Re') (Reflexivity) $x \leqslant x$,

(*) $x \rightarrow y=1 \Longrightarrow(z \rightarrow x) \rightarrow(z \rightarrow y)=1$,

(*) $x \leqslant y \Longrightarrow z \rightarrow x \leqslant z \rightarrow y$,

(**) $x \rightarrow y=1 \Longrightarrow(y \rightarrow z) \rightarrow(x \rightarrow z)=1$,

(**) $x \leqslant y \Longrightarrow y \rightarrow z \leqslant x \rightarrow z$,

(Tr) (Transitivity) $x \rightarrow y=1=y \rightarrow z \Longrightarrow x \rightarrow z=1$,

(Tr') (Transitivity) $x \leqslant y$ and $y \leqslant z \Longrightarrow x \leqslant z$.

Lemma 2.1. (See Proposition 2.1 and Theorem 2.7 of [3]) Let $\mathcal{A}=(A ; \rightarrow, 1)$ be an algebra of type $(2,0)$. Then the following hold:

(i) $(\mathrm{Re})+(\mathrm{Ex})+(\mathrm{An})$ imply $(\mathrm{M})$,

(ii) $(\mathrm{M})+(\mathrm{B})$ imply $(*),(* *)$,

(iii) $(\mathrm{M})+(* *)$ imply $(\operatorname{Tr})$,

(iv) $(\mathrm{Re})+(\mathrm{Ex})+(*)$ imply $(\mathrm{BB})$,

(v) $(\mathrm{M})+(\mathrm{BB})$ imply (B). 
Definition 2.2. (See [3]) An algebra $\mathcal{A}=(A ; \rightarrow, 1)$ is called:

1. RM algebra if it verifies the axioms: $(\mathrm{Re}),(\mathrm{M})$.

2. aRM algebra (or BH algebra) if it verifies the axioms: (Re), (M), (An), i.e., it is an RM algebra with (An).

3. RME algebra (or CI algebra) if it verifies the axioms: (Re), (M), (Ex), i.e., it is an RM algebra with (Ex).

4. $B C H$ algebra if it verifies the axioms: (Re), (Ex), (An), hence (M) by Lemma 2.1 (i), i.e., it is an RME algebra with (An).

5. $a R M^{* *}$ algebra if it verifies the axioms: $(\mathrm{Re}),(\mathrm{M}),(\mathrm{An}),\left({ }^{* *}\right)$, i.e., it is an aRM algebra with $(* *)$.

6. ${ }^{*} a R M^{* *}$ algebra if it verifies the axioms: (Re), (M), (An), $(*),\left({ }^{* *}\right)$, i.e., it is an aRM algebra with $\left(^{*}\right),\left({ }^{*}\right)$.

7. $B C H^{* *}$ algebra if it is a $\mathrm{BCH}$ algebra verifying $(* *)$.

8. $B C I$ algebra if it is a $\mathrm{BCH}$ algebra verifying (B).

Denote by $\mathbf{R M}, \mathbf{a R M}=\mathbf{B H}, \mathbf{R M E}=\mathbf{C I}, \mathbf{B C H}, \mathbf{a R M}^{* *},{ }^{*} \mathbf{a R M}^{* *}, \mathbf{B C H}^{* *}$, and $\mathbf{B C I}$ the classes of $\mathrm{RM}$, aRM, RME, BCH, aRM**, ${ }^{*} \mathrm{aRM}^{* *}, \mathrm{BCH}^{* *}$, and $\mathrm{BCI}$ algebras, respectively. From the definitions and Lemma 2.1(ii) it follows that

$$
\begin{gathered}
\mathrm{BCI} \subset \mathbf{B C H}^{* *} \subset \mathbf{B C H} \subset \mathbf{R M E} \subset \mathbf{R M}, \\
\mathbf{B C I} \subset \text { aRM }^{* *} \subset \mathbf{a R M}^{* *} \subset \mathbf{a R M} \subset \mathbf{R M}, \quad \text { and } \mathbf{B C H} \subset \mathbf{a R M} .
\end{gathered}
$$

The interrelationships between the classes of algebras mentioned before are visualized in Figure 1. (An arrow indicates proper inclusion, that is, if $\mathbf{X}$ and $\mathbf{Y}$ are classes of algebras, then $\mathbf{X} \longrightarrow \mathbf{Y}$ means $\mathbf{X} \subset \mathbf{Y}$.)

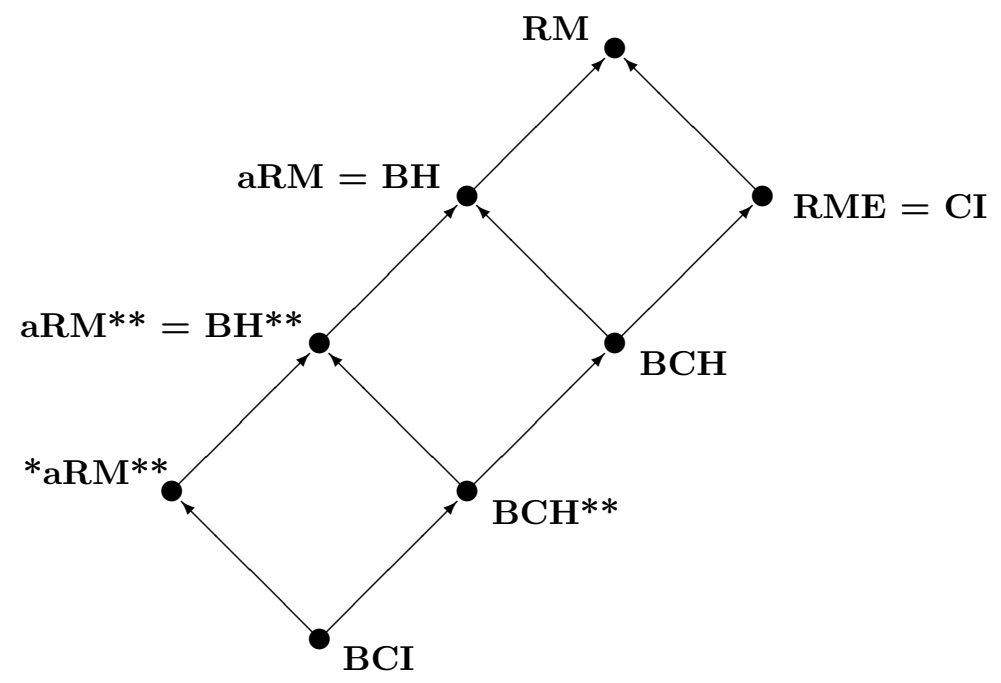

Fig. 1: The hierarchy between BCI and RM 
Note that, in RM algebras, $\leqslant$ is a reflexive relation; in aRM algebras, it is reflexive and antisymmetric. If $\mathcal{A}$ is an aRM** algebra, then $\leqslant$ is also transitive by Lemma 2.1(iii). Therefore $\leqslant$ is an order relation in aRM** algebras (hence also in $\mathrm{BCH}^{* *}, * \mathrm{aRM}^{* *}$, and $\mathrm{BCI}$ algebras).

\subsection{Generalizations of commutative groups}

A. Iorgulescu [5] introduced and studied new generalizations of groups such as moons, goops and many others.

Definition 2.3. A moon is an algebra $\mathcal{G}=\left(G ; \cdot,^{-1}, 1\right)$ of type $(2,1,0)$ satisfying

(U) $x \cdot 1=x=1 \cdot x$,

(Iv) $x \cdot x^{-1}=1=x^{-1} \cdot x$.

A moon is involutive if it satisfies

(DN) $\left(x^{-1}\right)^{-1}=x$.

A moon is associative if it satisfies

(Ass) $x \cdot(y \cdot z)=(x \cdot y) \cdot z$.

A moon is commutative if it satisfies

(Com) $x \cdot y=y \cdot x$.

Note that the associative moon is just the group.

Definition 2.4. ([5]) A goop is an algebra $\left(G ; \cdot,^{-1}, 1\right)$ of type $(2,1,0)$ satisfying $(\mathrm{U})$ and the following conditions:

(GP1) $y \cdot x^{-1}=1 \Longleftrightarrow x^{-1} \cdot y=1$,

(GP2) $y \cdot x^{-1}=1 \Longleftrightarrow x=y$.

Proposition 2.5. An algebra $\mathcal{G}=\left(G ; \cdot,{ }^{-1}, 1\right)$ of type $(2,1,0)$ is a goop if and only if it is an involutive moon satisfying

(GP) $y \cdot x^{-1}=1 \Longrightarrow x=y$.

Proof. Let $\mathcal{G}$ be a goop. Observe that it satisfies (Iv). Indeed, let $x \in G$. We have

$$
x=x \stackrel{(\mathrm{GP} 2)}{\Longleftrightarrow} x \cdot x^{-1}=1 \stackrel{(\mathrm{GP} 1)}{\Longleftrightarrow} x^{-1} \cdot x=1 .
$$

Therefore, (Iv) holds. By (Iv), $\left(x^{-1}\right)^{-1} \cdot x^{-1}=1$. From (GP2) we conclude that $\left(x^{-1}\right)^{-1}=x$. Thus $\mathcal{G}$ is an involutive moon. By (GP2), it satisfies (GP).

Conversely, let $\mathcal{G}$ satisfy (U), (Iv), (DN) and (GP). Let $x, y \in G$. To prove (GP1), we first assume that $y \cdot x^{-1}=1$. By $(\mathrm{GP}), x=y$. Then $x^{-1} \cdot y=x^{-1} \cdot x \stackrel{\text { (Iv) }}{=} 1$. Now suppose that $x^{-1} \cdot y=1$. Therefore, $1=x^{-1} \cdot y \stackrel{(\mathrm{DN})}{=} x^{-1} \cdot\left(y^{-1}\right)^{-1}$. Applying (GP), we see that $x^{-1}=y^{-1}$. Hence $y \cdot x^{-1}=y \cdot y^{-1}=1$. Consequently, (GP1) holds. Using (GP) and (Iv), we have (GP2). Thus, $\mathcal{G}$ is a goop. 
Definition 2.6. ([15]) We say that an algebra $\left(G ; \cdot{ }^{-1}, 1\right)$ of type $(2,1,0)$ is a weakly goop if it is an involutive moon satisfying

(wGP) $y \cdot x^{-1}=1=x \cdot y^{-1} \Longrightarrow x=y$.

Example 2.7. The algebra $\mathcal{G}=\left(\{a, b, 1\} ; \cdot{ }^{-1}, 1\right)$, with

\begin{tabular}{l|lll}
$\cdot$ & $a$ & $b$ & 1 \\
\hline$a$ & $a$ & 1 & $a$ \\
$b$ & 1 & 1 & $b$ \\
1 & $a$ & $b$ & 1
\end{tabular}

and $a^{-1}=b, b^{-1}=a, 1^{-1}=1$, is a commutative weakly goop. Since $b \cdot a^{-1}=$ $b \cdot b=1$ and $a \neq b, \mathcal{G}$ does not satisfy (GP2). Therefore, it is not a goop.

Let inv. com. moon, com. weakly goop, com. goop and com. group denote the class of all involutive commutative moons, commutative weakly goops, commutative goops and commutative groups, respectively. From the definitions we obtain

inv. com. moon $\subset$ com. weakly goop $\subset$ com. goop $\subset$ com. group

\section{The conditions $(\mathrm{p}-\mathrm{s}),(\mathrm{p}-\mathrm{s} 1),(\mathrm{D} 1=)$ and $(\mathrm{PS})$}

Let $\mathcal{A}=(A ; \rightarrow, 1)$ be an algebra of type $(2,0)$. Consider the following conditions that can be satisfied by $\mathcal{A}$ :

$$
\begin{aligned}
& (\mathrm{p}-\mathrm{s}) x \leqslant y \Longrightarrow x=y, \\
& (\mathrm{p}-\mathrm{s} 1) x \leqslant 1 \Longrightarrow x=1 \\
& (\mathrm{D} 1=) x=(x \rightarrow 1) \rightarrow 1, \\
& (\mathrm{PS})(x \rightarrow 1) \rightarrow y=(y \rightarrow 1) \rightarrow x .
\end{aligned}
$$

Note that, in [5], the concept of negation, ${ }^{-1}$, is defined by $x^{-1}=x \rightarrow 1$, and hence

$$
(\mathrm{D} 1=) \Longleftrightarrow\left(x^{-1}\right)^{-1}=x \text { and }(\mathrm{PS}) \Longleftrightarrow x^{-1} \rightarrow y=y^{-1} \rightarrow x .
$$

Thus $(\mathrm{D} 1=)$ is in fact the double negation property (DN) and (PS) is the property (pDNeg2), in the commutative case, from the book [5].

Remark that RM algebras satisfying (D1=) were studied in [15].

First we present connections between the conditions in the above list.

Lemma 3.1. Let $\mathcal{A}$ be an algebra of type $(2,0)$. Then

(i) $(\mathrm{M})+(\mathrm{PS})$ imply $(\mathrm{D} 1=)$,

(ii) $(\mathrm{M})+(\mathrm{D} 1=)$ imply $(\mathrm{p}-\mathrm{s} 1)$,

(iii) $(\mathrm{An})+(\mathrm{Re})+\left({ }^{* *}\right)+(\mathrm{p}-\mathrm{s} 1)$ imply $(\mathrm{p}-\mathrm{s})$, 
(iv) $(\mathrm{D} 1)+(\mathrm{p}-\mathrm{s})$ imply $(\mathrm{D} 1=)$,

(v) $(\operatorname{Re})+(\mathrm{Ex})+(\mathrm{D} 1=)$ imply $(\mathrm{p}-\mathrm{s})$.

(vi) (p-s) implies (An), (*), (**).

Proof. (i) Let $x \in A$. By (PS) and (M), $(x \rightarrow 1) \rightarrow 1=(1 \rightarrow 1) \rightarrow x=x$, that is, $(\mathrm{D} 1=)$ holds.

(ii) Let $x \in A$ and $x \leqslant 1$. Hence $x \rightarrow 1=1$. From (D1=) we have $x=(x \rightarrow$ 1) $\rightarrow 1=1 \rightarrow 1=1$. Consequently, $\mathcal{A}$ satisfies (p-s1).

(iii) Let $x, y \in A$ and $x \leqslant y$. By (**) and (Re), $y \rightarrow x \leqslant x \rightarrow x=1$. From (p-s1) we obtain $y \rightarrow x=1$, that is, $y \leqslant x$. Applying (An), we conclude that $x=y$. Then (p-s) holds.

(iv) This is immediate.

(v) Let $x, y \in A$ and suppose that $x \leqslant y$. Then $x \rightarrow y=1$. Applying (Ex) and (Re), we get

$$
y \rightarrow 1=y \rightarrow(x \rightarrow y)=x \rightarrow(y \rightarrow y)=x \rightarrow 1 .
$$

Therefore, $x=(x \rightarrow 1) \rightarrow 1=(y \rightarrow 1) \rightarrow 1=y$ by (D1=). Thus ( $\mathrm{p}-\mathrm{s})$ is satisfied.

(vi) Obvious.

Definition 3.2. An $\mathrm{RM}$ algebra $\mathcal{A}=(A ; \rightarrow, 1)$ is $p$-semisimple ([5]) if (p-s) holds in $\mathcal{A}$.

Remark 3.3. From Lemma 3.1 (vi) it follows that p-semisimple RM algebras coincide with p-semisimple aRM algebras, with p-semisimple $\mathrm{aRM}^{* *}$ algebras, and with p-semisimple ${ }^{*} \mathrm{aRM}^{* *}$ algebras. Moreover, p-semisimple RME algebras coincide with $\mathrm{p}$-semisimple $\mathrm{BCH}$ algebras, with $\mathrm{p}$-semisimple $\mathrm{BCH}^{* *}$ algebras, and with p-semisimple BCI algebras.

Example 3.4. Consider the set $A=\{a, b, 1\}$ with the following table of $\rightarrow$ :

\begin{tabular}{c|ccc}
$\rightarrow$ & $a$ & $b$ & 1 \\
\hline$a$ & 1 & $b$ & $a$ \\
$b$ & $a$ & 1 & $a$ \\
1 & $a$ & $b$ & 1
\end{tabular}

The properties $(\mathrm{Re}),(\mathrm{M}),(\mathrm{An}),\left({ }^{*}\right),\left({ }^{* *}\right)$ and $(\mathrm{p}-\mathrm{s})$ are satisfied. The algebra $\mathcal{A}=(A ; \rightarrow, 1)$ does not satisfy (Ex) for $x=a, y=b, z=1$. Therefore, $\mathcal{A}$ is a p-semisimple $* \mathrm{aRM}^{* *}$ algebra, which is not an RME algebra (hence, it is also not a BCI algebra).

From Lemma 3.1(i) and (ii) we have

Lemma 3.5. If an algebra $\mathcal{A}$ verifies $(\mathrm{M})$, then

$$
(\mathrm{PS}) \Longrightarrow(\mathrm{D} 1=) \Longrightarrow(\mathrm{p}-\mathrm{s} 1) \text {. }
$$


Proposition 3.6. Let $\mathcal{A}$ be an aRM** algebra satisfying (D1). Then

$$
(\mathrm{p}-\mathrm{s} 1) \Longleftrightarrow(\mathrm{p}-\mathrm{s}) \Longleftrightarrow(\mathrm{D} 1=)
$$

Proof. (p-s1) $\Longrightarrow$ (p-s) By Lemma 3.1 (iii).

$(\mathrm{p}-\mathrm{s}) \Longrightarrow(\mathrm{D} 1=)$ Follows from Lemma 3.1 (iv).

$(\mathrm{D} 1=) \Longrightarrow(\mathrm{p}-\mathrm{s} 1)$ Obviously, by Lemma 3.1 (ii).

Proposition 3.7. ([16]) Let $\mathcal{A}$ be a BCI algebra. Then

$$
(\mathrm{p}-\mathrm{s}) \Longleftrightarrow(\mathrm{p}-\mathrm{s} 1) \Longleftrightarrow(\mathrm{D} 1=) \Longleftrightarrow(\mathrm{PS}) \text {. }
$$

We introduced now the following definition.

Definition 3.8. Let $\mathcal{A}=(A ; \rightarrow, 1)$ be an algebra of type $(2,0)$.

9. $\mathcal{A}$ is an $R M(P S)$ algebra if it is an RM algebra verifying (PS).

10. $\mathcal{A}$ is an $a R M(P S)$ algebra (resp. $a R M^{* *}(P S),{ }^{*} a R M^{* *}(P S)$ algebra) if it is an aRM algebra (resp. aRM ${ }^{* *},{ }^{*} \mathrm{aRM}^{* *}$ algebra) verifying (PS).

11. $\mathcal{A}$ is an $R M E(P S)$ algebra (resp. $B C H(P S), B C H^{* *}(P S)$, and $B C I(P S)$ algebra) if it is an RME algebra (resp. $\mathrm{BCH}, \mathrm{BCH}^{* *}$, and $\mathrm{BCI}$ algebra) verifying (PS).

Remark 3.9. From Lemma 3.1 (i)-(iii) we see that every ${ }^{*} a \mathrm{RM}^{* *}(\mathrm{Ps})$ algebra is p-semisimple. The converse is not true, that is, there is a p-semisimple ${ }^{*} \mathrm{aRM}^{* *}$ algebra that does not satisfy (PS); see Example 3.4.

Proposition 3.10. Let $\mathcal{A}=(A ; \rightarrow, 1)$ be an algebra of type $(2,0)$. The following are equivalent:

(i) $\mathcal{A}$ is a $B C H(P S)$ algebra,

(ii) $\mathcal{A}$ is an $R M E(P S)$ algebra,

(iii) $\mathcal{A}$ is a $B C H^{* *}(P S)$ algebra,

(iv) $\mathcal{A}$ is a $B C I(P S)$ algebra,

(v) $\mathcal{A}$ is a p-semisimple BCI algebra.

Proof. (i) $\Rightarrow$ (ii) This is obvious.

(ii) $\Rightarrow$ (iii) Let $\mathcal{A}$ verify (Re), (M), (Ex) and (PS). From Lemma 3.1 (i) and (v) we deduce that $\mathcal{A}$ satisfies (p-s). Hence, by Lemma 3.1 (vi), (An) and (**) hold in $\mathcal{A}$. Thus, $\mathcal{A}$ is a $\mathrm{BCH}^{* *}(\mathrm{Ps})$ algebra.

(iii) $\Rightarrow$ (iv) By Lemma 3.1 (i)-(iii) we conclude that $\mathcal{A}$ satisfies (p-s). From (p-s) it follows that $\mathcal{A}$ also satisfies $(*)$. Applying Lemma 2.1 (iv) and (v) we see that (B) holds in $\mathcal{A}$. Consequently, $\mathcal{A}$ is a BCI algebra.

(iv) $\Rightarrow$ (v) By Remark 3.9.

(v) $\Rightarrow$ (i) This follows from Proposition 3.7. 
Proposition 3.11. Let $\mathcal{A}=(A ; \rightarrow, 1)$ be an algebra of type $(2,0)$. Then $\mathcal{A}$ is an $a R M^{* *}(P S)$ algebra if and only if it is an *aRM**(PS) algebra.

Proof. Let $\mathcal{A}$ be an $\left.\operatorname{RRM}^{* *}{ }_{(P S}\right)$ algebra. Then $\mathcal{A}$ satisfies $(\mathrm{An}),(\operatorname{Re}),(\mathrm{M}),\left({ }^{* *}\right)$, (PS). Applying Lemma 3.1 (i)-(iii) and (vi) we deduce that $\left(^{*}\right)$ holds in $\mathcal{A}$. Thus $\mathcal{A}$ is an ${ }^{*} \mathrm{RM}^{* *}(P S)$ algebra. The converse is obvious.

Let us denote by RM(ps), aRM(ps), aRM**(ps), *aRM**(ps), RME(ps), BCH(ps), $\mathbf{B C H}^{* *}(\mathrm{ps})$, and BCI(ps) the classes of RM(ps), aRM(ps), aRM**(ps), ${ }^{*} \mathrm{aRM}^{* *}(\mathrm{Ps}), \mathrm{RME}(\mathrm{Ps}), \mathrm{BCH}(\mathrm{PS}), \mathrm{BCH}^{* *}(\mathrm{Ps})$, and $\mathrm{BCI}(\mathrm{Ps})$ algebras, respectively.

From Propositions 3.10 and 3.11 it follows that

$$
\begin{gathered}
\mathrm{RME}(\mathrm{Ps})=\mathrm{BCH}(\mathrm{Ps})=\mathrm{BCH}^{* *}(\mathrm{Ps})=\mathrm{BCI}(\mathrm{Ps})=\mathrm{p}-\mathrm{s}-\mathrm{BCI} \text { and } \\
\mathrm{aRM}^{* *}(\mathrm{Ps})=* \mathrm{aRM}^{* *}(\mathrm{Ps}),
\end{gathered}
$$

where $\mathbf{p - s - B C I}$ denotes the class of all p-semisimple BCI algebras.

Furthermore, there are inclusions:

$$
\text { p-s-BCI } \subset{ }^{*} \mathrm{aRM}^{* *}(\mathrm{Ps}) \subset \mathrm{aRM}(\mathrm{Ps}) \subset \mathrm{RM}(\mathrm{Ps}) .
$$

These inclusions are proper, see examples below.

Example 3.12. Consider the set $A=\{a, b, 1\}$ and the operation $\rightarrow$ given by the following table:

\begin{tabular}{l|lll}
$\rightarrow$ & $a$ & $b$ & 1 \\
\hline$a$ & 1 & $b$ & $b$ \\
$b$ & $b$ & 1 & $a$ \\
1 & $a$ & $b$ & 1
\end{tabular}

It is easy to see that the properties $(\mathrm{An}),(\mathrm{Re}),(\mathrm{M}),\left({ }^{*}\right),\left({ }^{* *}\right)$ and $(\mathrm{PS})$ are satisfied; $(\mathrm{Ex})$ is not satisfied for $(x, y, z)=(a, b, a)$. Hence, $\mathcal{A}=(A ; \rightarrow, 1)$ is an ${ }^{*} \mathrm{aRM}^{* *}(\mathrm{Ps})$ algebra, which is not a BCI algebra.

Example 3.13. Let $A=\{a, b, 1\}$ and $\rightarrow$ be defined as follows:

\begin{tabular}{l|lll}
$\rightarrow$ & $a$ & $b$ & 1 \\
\hline$a$ & 1 & 1 & $b$ \\
$b$ & $a$ & 1 & $a$ \\
1 & $a$ & $b$ & 1
\end{tabular}

The algebra $\mathcal{A}=(A ; \rightarrow, 1)$ satisfies properties (An), (Re), (M), (PS). It does not satisfy $\left({ }^{*}\right)$ for $(x, y, z)=(b, a, b),\left({ }^{* *}\right)$ for $(x, y, z)=(a, a, b)$. Then $\mathcal{A}$ is an $\operatorname{aRM}(\mathrm{Ps})$ algebra, which is not an ${ }^{*} \mathrm{aRM}^{* *}$ algebra. 
Example 3.14. Consider the set $A=\{a, b, c, 1\}$ and the operation $\rightarrow$ given by the following table:

\begin{tabular}{c|cccc}
$\rightarrow$ & $a$ & $b$ & $c$ & 1 \\
\hline$a$ & 1 & 1 & $b$ & $a$ \\
$b$ & 1 & 1 & $c$ & $b$ \\
$c$ & $b$ & $c$ & 1 & $c$ \\
1 & $a$ & $b$ & $c$ & 1
\end{tabular}

Then the properties (Re), (M), (PS) are satisfied. (An) is not satisfied for $(x, y)=$ $(a, b)$. Therefore, $\mathcal{A}=(A ; \rightarrow, 1)$ is an $\mathrm{RM}(\mathrm{Ps})$ algebra, which is not an aRM algebra.

\section{4. $\mathrm{RM}(\mathrm{Ps})$ algebras and involutive commutative moons}

In this section, we establish the connections between:

- $\mathrm{RM}(P S)$ algebras and involutive commutative moons,

- $\operatorname{aRM}(P S)$ algebras and commutative weakly goops,

— ${ }^{*} \mathrm{aRM}^{* *}(P S)$ algebras and commutative goops.

Note that connections between goops and implicative-goops are presented in Chapter 12 of [5].

Let $\mathcal{A}=(A ; \rightarrow, 1)$ be an algebra of type $(2,0)$. Define $\Phi(\mathcal{A})=\left(A ; \cdot{ }^{-1}, 1\right)$ by: for all $x, y \in A, x \cdot y=(y \rightarrow 1) \rightarrow x$ and $x^{-1}=x \rightarrow 1$.

Let $\mathcal{G}=\left(G ; \cdot{ }^{-1}, 1\right)$ be an algebra of type $(2,1,0)$. Define $\Psi(\mathcal{G})=(G ; \rightarrow, 1)$ by: for all $x, y \in G, x \rightarrow y=y \cdot x^{-1}$.

Lemma 4.1. Let $\mathcal{A}=(A ; \rightarrow, 1)$ be an algebra of type $(2,0)$. Then

(i) $(\mathrm{M})$ and $(\mathrm{D} 1=)$ imply $(\mathrm{U})$ in $\Phi(\mathcal{A})$,

(ii) $(\mathrm{Re})$ and $(\mathrm{D} 1=)$ imply (Iv) in $\Phi(\mathcal{A})$,

(iii) $(\mathrm{D} 1=)$ implies $(\mathrm{DN})$ in $\Phi(\mathcal{A})$,

(iv) (An) implies (wGP) in $\Phi(\mathcal{A})$,

(v) (Re), (D1=), and (p-s) imply (GP) in $\Phi(\mathcal{A})$,

(vi) (M) and (PS) imply (Com) in $\Phi(\mathcal{A})$.

Proof. (i) Let $x \in A$. By (M), $x \cdot 1=(1 \rightarrow 1) \rightarrow x=x$. Applying (D1=), we get $1 \cdot x=(x \rightarrow 1) \rightarrow 1=x$. Therefore, $\Phi(\mathcal{A})$ satisfies $(\mathrm{U})$.

(ii) From $(\mathrm{D} 1=)$ and $(\mathrm{Re})$ we obtain

$$
\begin{gathered}
x \cdot x^{-1}=((x \rightarrow 1) \rightarrow 1) \rightarrow x=x \rightarrow x=1 \text { and } \\
x^{-1} \cdot x=(x \rightarrow 1) \rightarrow(x \rightarrow 1)=1 .
\end{gathered}
$$

Then (Iv) holds in $\Phi(\mathcal{A})$.

(iii) and (iv) are obvious. 
(v) To prove (GP), let $x, y \in A$ and $y \cdot x^{-1}=1$. Then $x \rightarrow y=((x \rightarrow 1) \rightarrow 1) \rightarrow$ $y=y \cdot x^{-1}=1$. Hence $x \leqslant y$. By (p-s), $x=y$. Therefore (GP) holds in $\Phi(\mathcal{A})$.

(vi) Let (M) and (PS) are satisfied and let $x, y \in A$. From Lemma 3.1(i) we obtain $(\mathrm{D} 1=)$. Consequently, $(\mathrm{DN})$ holds in $\Phi(\mathcal{A})$. We have

$$
x \cdot y \stackrel{(\mathrm{DN})}{=} x \cdot\left(y^{-1}\right)^{-1}=y^{-1} \rightarrow x=(y \rightarrow 1) \rightarrow x \stackrel{(\mathrm{PS})}{=}(x \rightarrow 1) \rightarrow y=y \cdot x,
$$

that is, $(\mathrm{Com})$ is satisfied in $\Phi(\mathcal{A})$.

Lemma 4.2. Let $\mathcal{G}=\left(G ; \cdot,^{-1}, 1\right)$ be an algebra of type $(2,1,0)$. Then

(i) (Iv) implies (Re) in $\Psi(\mathcal{G})$,

(ii) (U) and (Iv) imply (M) in $\Psi(\mathcal{G})$,

(iii) (Com) and (DN) imply (PS) in $\Psi(\mathcal{G})$,

(iv) (wGP) implies (An) in $\Psi(\mathcal{G})$.

Proof. (i) Let $x \in G$. By (Iv), $x \rightarrow x=x \cdot x^{-1}=1$, that is, (Re) holds in $\Psi(\mathcal{G})$.

(ii) Applying (U) and (Iv), we get

$$
1 \rightarrow x=x \cdot 1^{-1}=x \cdot\left(1 \cdot 1^{-1}\right)=x \cdot 1=x,
$$

i.e., $\Psi(\mathcal{G})$ satisfies $(\mathrm{M})$.

(iii) Let $x, y \in G$. We have

$$
(x \rightarrow 1) \rightarrow y=y \cdot\left(x^{-1}\right)^{-1} \stackrel{(\mathrm{DN})}{=} y \cdot x \stackrel{(\mathrm{Com})}{=} x \cdot y=(y \rightarrow 1) \rightarrow x,
$$

that is, (PS) holds in $\Psi(\mathcal{G})$.

(iv) is immediate.

Theorem 4.3. $\quad(\mathrm{i})$ Let $\mathcal{A}=(A ; \rightarrow, 1)$ is an $R M(P S)$ algebra. Then $\Phi(\mathcal{A})=$ $\left(A ; \cdot,{ }^{-1}, 1\right)$ is an involutive commutative moon.

(ii) Let $\mathcal{G}=\left(G ; \cdot,{ }^{-1}, 1\right)$ is an involutive commutative moon. Then $\Psi(\mathcal{G})=$ $(G ; \rightarrow, 1)$ is an $R M(P S)$ algebra.

(iii) Given $\mathcal{A}$ and $\mathcal{G}$ as above we have $\Psi \Phi(\mathcal{A})=\mathcal{A}$ and $\Phi \Psi(\mathcal{G})=\mathcal{G}$.

Proof. (i) Let $\mathcal{A}$ be an $\mathrm{RM}(\mathrm{Ps})$ algebra. Then $\mathcal{A}$ satisfies (Re), (M), (PS) and, by Lemma 3.1(i), also satisfies (D1=). From Lemma 4.1 it follows that (U), (Iv), (DN) and $(\mathrm{Com})$ hold in $\Phi(\mathcal{A})$. Therefore, $\Phi(\mathcal{A})$ is an involutive commutative moon.

(ii) Let $\mathcal{G}$ verify $(\mathrm{U}),(\mathrm{Iv}),(\mathrm{DN})$ and $(\mathrm{Com})$. By Lemma 4.2(i)-(iii), $\Psi(\mathcal{G})$ verifies (Re), (M) and (PS). Hence $\Psi(\mathcal{G})$ is an $\mathrm{RM}(\mathrm{Ps})$ algebra.

(iii) Suppose $\mathcal{A}=(A ; \rightarrow, 1)$ is an $\operatorname{RM}(\mathrm{ps})$ algebra and $x \in A$. Then, in $\Phi(\mathcal{A})=$ $\left(A ; \cdot,^{-1}, 1\right)$, we obtain $x^{-1}=x \rightarrow 1$ and

$$
y \cdot x^{-1}=y \cdot(x \rightarrow 1)=((x \rightarrow 1) \rightarrow 1) \rightarrow y=x \rightarrow y .
$$

Thus $\Psi \Phi(\mathcal{A})=\mathcal{A}$. 
Next suppose $\mathcal{G}=\left(G ; \cdot{ }^{-1}, 1\right)$ is an involutive moon and $x, y \in G$. Then, in $\Psi(\mathcal{G})=(G ; \rightarrow, 1)$, we get $x \rightarrow 1=1 \cdot x^{-1}=x^{-1}$ and

$$
(y \rightarrow 1) \rightarrow x=y^{-1} \rightarrow x=x \cdot\left(y^{-1}\right)^{-1}=x \cdot y .
$$

Thus $\Phi \Psi(\mathcal{G})=\mathcal{G}$.

Hence, by above Theorem 4.3, we have the equivalence

$$
\mathrm{RM}(\mathrm{PS}) \Longleftrightarrow \text { inv. com. moon. }
$$

Theorem 4.4. (i) Let $\mathcal{A}=(A ; \rightarrow, 1)$ be an aRM(PS) algebra ( ${ }^{*} a R M^{* *}(P S)$ algebra). Then $\Phi(\mathcal{A})=\left(A ; \cdot,{ }^{-1}, 1\right)$ is a commutative weakly goop (commutative goop, respectively).

(ii) Let $\mathcal{G}=\left(G ; \cdot,^{-1}, 1\right)$ be a commutative weakly goop (commutative goop). Then $\Psi(\mathcal{G})=(G ; \rightarrow, 1)$ is an aRM(PS) algebra $\left({ }^{*} a R M^{* *}(P S)\right.$ algebra, respectively).

(iii) Given $\mathcal{A}$ and $\mathcal{G}$ as above we have $\Psi \Phi(\mathcal{A})=\mathcal{A}$ and $\Phi \Psi(\mathcal{G})=\mathcal{G}$.

Proof. (i) Let $\mathcal{A}$ be an aRM(Ps) algebra. By Theorem 4.3, $\Phi(\mathcal{A})$ is an involutive commutative moon. Since (An) implies (wGP) (by Lemma 4.1(iv)), $\Phi(\mathcal{A})$ is a weakly goop. Therefore, it is a commutative weakly goop. If $\mathcal{A}$ is an ${ }^{*} \mathrm{aRM}{ }^{* *}(\mathrm{ps})$ algebra then, by Lemma 3.1(i)-(iii), $\mathcal{A}$ satisfies (p-s). From Lemma 4.1(v) we see that (GP) holds in $\Phi(\mathcal{A})$. Thus $\Phi(\mathcal{A})$ is a goop by Proposition 2.5. Finally, $\Phi(\mathcal{A})$ is a commutative goop.

(ii) Let $\mathcal{G}$ be a commutative weakly goop. Since $\mathcal{G}$ is an involutive commutative moon, $\Psi(\mathcal{G})$ is an $\operatorname{RM}(\mathrm{Ps})$ algebra by Theorem 4.3. From Lemma 4.2 (iv) we deduce that (An) holds in $\Psi(\mathcal{G})$. Thus $\Psi(\mathcal{G})$ is an $\operatorname{aRM}(\mathrm{Ps})$ algebra.

Now let $\mathcal{G}$ be a commutative goop. From (GP) it follows that (p-s) holds in $\Psi(\mathcal{G})$. Hence, $\Psi(\mathcal{G})$ satisfies $(*)$ and $(* *)$. Thus $\Psi(\mathcal{G})$ is an $\operatorname{aRM}^{* *}(\mathrm{PS})$ algebra.

(iii) See the proof of Theorem 4.3 (iii).

Hence, by above Theorem 4.4, we have the equivalences:

$\operatorname{aRM}(\mathrm{PS}) \Longleftrightarrow$ com. weakly goop and ${ }^{*} \mathrm{aRM}^{* *}(\mathrm{Ps}) \Longleftrightarrow$ com. goop.

Theorem 4.5. ([9]) If $\mathcal{A}=(A ; \rightarrow, 1)$ is a p-semisimple $B C I$ algebra (or, equivalently, $B C H^{* *}(P S)$ algebra), then $\Phi(\mathcal{A})=\left(A ; \cdot,{ }^{-1}, 1\right)$ is a commutative group. Conversely, if $\mathcal{G}=\left(G ; \cdot{ }^{-1}, 1\right)$ is a commutative group, then $\Psi(\mathcal{G})=(G ; \rightarrow, 1)$ is a p-semisimple BCI algebra.

Hence, by Theorem 4.5, we have the equivalence

$$
\text { p-s-BCI }=\text { BCH }^{* *}(\mathrm{Ps})=\mathrm{BCH}(\mathrm{Ps})=\mathrm{RME}(\mathrm{Ps}) \Longleftrightarrow \text { com. group. }
$$

Acknowledgement. The author would like to thank the referee for the valuable suggestions and comments. 


\section{References}

[1] M. Aslam and A. B. Thaheem, A note on p-semisimple BCI-algebras, Math. Japon., 36 (1991), 39-45.

[2] Q. P. Hu and X. Li, On BCH-algebras, Math. Sem. Notes Kobe Univ., 11 (1983), 313-320.

[3] A. Iorgulescu, New generalizations of BCI, BCK and Hilbert algebras - Part I, J. Mult.-Valued Logic Soft Comput., 27(4) (2016), 353-406.

[4] A. Iorgulescu, New generalizations of BCI, BCK and Hilbert algebras - Part II, J. Mult.-Valued Logic Soft Comput., 27(4) (2016), 407-456.

[5] A. Iorgulescu, Implicative-Groups vs. Groups and Generalizations, Matrix Rom, Bucharest, 2018.

[6] K. Iséki, An algebra related with a propositional calculus, Proc. Japan. Acad., 42 (1966), 26-29.

[7] Y. B. Jun, E. H. Roh and H. S. Kim, On BH-algebras, Sci. Math., 1(3) (1998), 347-354.

[8] H. S. Kim and H. G. Park, On 0-commutative B-algebras, Sci. Math. Jpn., 62(1) (2005), 7-12, e-2005: 31-36.

[9] T. Lei and C. Xi, p-radical in BCI-algebras, Math. Japon., 30 (1985), 511-517.

[10] D. J. Meng, BCI-algebras and abelian groups, Math. Japon., 32 (1987), 693696.

[11] B. L. Meng, CI-algebras, Sci. Math. Jpn., 71 (2010), 11-17; e-2009: 695-701.

[12] A. Walendziak, Deductive systems and congruences in RM algebras, J. Mult.Valued Logic Soft Comput., 30 (2018), 521-539.

[13] A. Walendziak, The implicative property for some generalizations of BCK algebras, J. Mult.-Valued Logic Soft Comput., 31 (2018), 591-611.

[14] A. Walendziak, The property of commutativity for some generalizations of BCK algebras, Soft Comput., 23 (2019), 7505-7511.

[15] A. Walendziak, Some generalizations of p-semisimple BCI algebras and groups, Soft Comput., 24 (2020), 12781-12787.

[16] Q. Zhang, Some other characterizations of p-semisimple BCI-algebras, Math. Japon., 36 (1991), 815-817.

\section{Andrzej Walendziak}

Siedlce University of Natural Sciences and Humanities

Faculty of Exact and Natural Sciences

3 Maja 54, PL-08110 Siedlce, Poland

e-mail: walent@interia.pl 